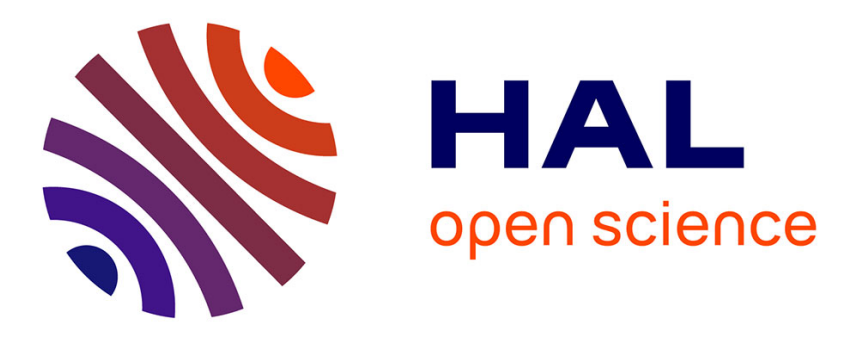

\title{
Investigation of the collapse of bubbles after the impact of a piston on a liquid free surface
}

\author{
Maya Mounir Daou, Elena Igualada, Hugo Dutilleul, Jean-Marie Citerne, \\ Javier Rodríguez-Rodríguez, Stéphane Zaleski, Daniel Fuster
}

\section{- To cite this version:}

Maya Mounir Daou, Elena Igualada, Hugo Dutilleul, Jean-Marie Citerne, Javier Rodríguez-Rodríguez, et al.. Investigation of the collapse of bubbles after the impact of a piston on a liquid free surface. AIChE Journal, 2017, 63 (6), pp.2483-2495 10.1002/aic.15725 . hal-01510299

\section{HAL Id: hal-01510299 \\ https: / hal.sorbonne-universite.fr/hal-01510299}

Submitted on 19 Apr 2017

HAL is a multi-disciplinary open access archive for the deposit and dissemination of scientific research documents, whether they are published or not. The documents may come from teaching and research institutions in France or abroad, or from public or private research centers.
L'archive ouverte pluridisciplinaire HAL, est destinée au dépôt et à la diffusion de documents scientifiques de niveau recherche, publiés ou non, émanant des établissements d'enseignement et de recherche français ou étrangers, des laboratoires publics ou privés. 


\title{
Investigation of the collapse of bubbles after the impact of a piston on a liquid free surface
}

\author{
Maya Mounir Daou ${ }^{1,2}$, Elena Igualada ${ }^{3}$, Hugo Dutilleul $^{1}$, Jean-Marie Citerne $^{1}$, \\ Javier Rodríguez-Rodríguez ${ }^{3}$, Stephane Zaleski ${ }^{1}$, Daniel Fuster ${ }^{1}{ }^{*}$ \\ ${ }^{1}$ Sorbonne Universités, UPMC Univ Paris 06, CNRS, UMR 7190, Institut Jean Le Rond \\ d'Alembert, F-75005, Paris, France \\ ${ }^{2}$ Sorbonne Universités, UPMC Univ Paris 06, CNRS, UMR 7197, Laboratoire de Réactivité \\ de Surface, F-75005, Paris, France \\ ${ }^{3}$ Fluid Mechanics Group, Universidad Carlos III de Madrid, Avd. Universidad 30, 28911 \\ Leganés, Madrid, Spain
}

\begin{abstract}
We describe a novel technique based on the impact of a piston on a liquid confined in a vessel. Pressure measurements reveal that strong pressure variations (up to 100 atmospheres) with a rich content of frequencies are efficiently transmitted to the liquid. High-speed camera visualizations show that pre-existing millimetric bubbles always collapse during the first instants of the impact whereas the behavior of submillimetric bubbles depends on the features of the pressure evolution in the system. In addition to the impact velocity, the amount of

* Corresponding author: fuster@dalembert.upmc.fr
\end{abstract}


gas/vapor trapped between the piston and the liquid's surface plays an important role on how pressure evolves. Only when negative pressure occurs bubbles grow significantly and collapse. The violent collapse of bubbles promote turbulence and mixing at very small length-scales which renders this technique interesting to intensify processes limited by heat and mass diffusion.

Keywords: Micromixing; Process intensification; Cavitation; Bubble collapse; Multiphase reactor

\section{Introduction}

Acoustic and hydrodynamic cavitation have been the object of intense research from a fundamental ${ }^{1 / 2}$ and an industrial perspective $\mathrm{e}^{3 / 7}$. Whether using ultrasound or hydraulic machineries, the basic mechanisms leading to the intense collapse of bubbles are similar ${ }^{6}$. After the system's pressure is decreased at constant temperature, vapor cavities appear inside the liquid. This process is usually accompanied by a violent agitation of the liquid due to a rapid formation and collapse of bubbles generating locally very high temperatures and pressures ${ }^{8}$. These two major and most widespread techniques have limitations ${ }^{9}$. Acoustic cavitation can be promoted by exciting a single or multiple frequencies in the range of $10 \mathrm{kHz}$ to $10 \mathrm{MHz}$. For instance, Hung and Hoffmann 10 used acoustic cavitation with frequencies ranging from 20 to $1078 \mathrm{kHz}$ to treat waste-water volumes from 90 up to 600 $\mathrm{mL}$ (depending on the frequency tested). Specially at very high frequencies, this technique is uneconomical for industrial reactors ${ }^{9111}$ due to the various 
problems arising from the significant attenuation of bubbly systems to the high erosion rates of the transducer surface and the economic viability of the process. Hydrodynamic cavitation is believed to present a better energy efficiency than techniques based on ultrasounds. However, its large scale application remains challenging since it modifies the flow field and the turbulence characteristics ${ }^{9[12}$ making this type of cavitation very difficult to scale-up and control. Thus, although many industrial processes are based on cavitation (i.e. semiconductor, leather processing and food industry) the two main problems of the currently used techniques, namely, the lack of reproducibility and control of the cavitation process and second, the upscaling of these technologies, restrain the efficiency and widespread application of these technologies.

The appearance and later collapse of bubbles can be triggered by mechanisms different than those typically used in ultrasonic and hydrodynamic cavitation. One example is the generation of bubbles induced from the response of a liquid or solid to a sudden impact. In solid mechanics e.g. spallation or dynamic fracture, cavitation occurs upon the impact of a projectile or a flyer on a target $13-15$. The first to investigate spalling was Hopkinson at the beginning of the previous century 16 . The basic principle is to impact a medium with a projectile or a flyer. After the reflection of the initial compression wave a tensile stress wave is generated in the medium and when the local tensile strength is larger than the strength of the material, small defects present in the solid structure are activated in a process similar to that of cavitation in liquids when the pressure falls below Blake's thresh- 
old $\sqrt{17-22}$. In this context, spallation refers to the ejection or vaporization of a material from the target in response to an impact. In liquids, the impact of a projectile flying across a vessel full of liquid is known to induce the formation of bubbles and its latter collapse 23 .

For instance, Dear and Field ${ }^{24}$ have used the "tube arrest" method 25 to study both the collapse of a single cavity and an array of cavities contained between two transparent plates after the impact of a slider 26 . Using a water/gelatine mixture, they were able to control several parameters such as the shape and the size of the bubbles $24 \mid 27$. Other relevant works include those of Daily et al. $\stackrel{28}{ }$, who studied the catastrophic rupture of glass bottles induced by the collapse of bubbles after an impact. Rodríguez-Rodríguez et al. ${ }^{29}$ report experiments on the dynamic response of bubbles in supersaturated media triggered by the impact on a vessel and Dular and CoutierDelgosha $^{30}$ use the "tube arrest" method to investigate the thermodynamic effects associated to the collapse of a single bubble.

In this work, we examine the phenomena induced by the impact of a piston on a liquid containing bubbles of controllable size confined in a closed vessel ${ }^{31}$. This device is designed to overcome the classical problems of acoustic and hydrodynamic cavitation. We show that using the plate impact method, we are capable of exciting bubbles of a wide size range in a large liquid volume. This is due to the efficient transmission of high amplitude pressure pulses with a low frequency content to the medium. The system is designed in order to control several variables that have proven to have 
a great influence on the bubble collapse process such as the bubble radius distribution and the frequency of the excitation wave. The control of the bubble collapse characteristics will be useful for future investigations on physical and chemical phenomena influenced by this phenomenon $32 \sqrt[35]{35}$.

The manuscript is structured as follows. First, we present the experimental device and we provide details about the methods used to obtain the experimental measurements. Then, we investigate the dynamics of the impact of a piston on a liquid free surface enclosed in a chamber. Finally the dynamic response of pre-existing bubbles to the impact is discussed.

\section{Experimental setup and measurements}

In this section we present the experimental set-up along with the velocity and pressure measurement systems. The experimental arrangement is schematically presented in Figure 1.

The apparatus consist in a cylindrical reactor (stainless steel, internal diameter $0.075 \mathrm{~m}$ ) filled with a liquid. The initial height of the liquid level, $H_{0,1}$ is typically of the order of $30 \mathrm{~cm}$. An upper piston of mass $m_{\mathrm{p}}=0.530 \mathrm{~kg}$ is used to impact the liquid free surface. The side walls of the reactor are equipped with two opposed optical accesses for the purpose of visualizing the liquid's response to the impact. The bottom part of the reactor consists in another piston whose displacement is restricted between two vertical po- 
sitions of separation length $d$. Note that when $d=0$, the reactor can be considered as a non-deformable rigid-wall vessel.

The initial height of the upper piston above the liquid free surface, $H_{0}$ is set by an electrical actuator which controls the displacement of an electromagnet. The attachment and release sequences of the piston by the electromagnet are controlled by a computer.

The initial pressure between the free surface of the liquid and the upper piston when the latter is still attached to the electromagnet, $P_{\text {top }, 0}$, is regulated using a vacuum pump. The minimum reachable value of $P_{\mathrm{top}, 0}$ is the liquid's vapor pressure, i.e. water in the experiments herein. $P_{\text {top }, 0}$ is measured using a digital vacuometer (LEO 2, Keller) attached to an evacuation pipe. It has a dynamic range from -0.1 to $0.3 \mathrm{MPa}$ with a $100 \mathrm{~Pa}$ resolution. Because of the unavoidable presence of dissolved gases in the liquid, $P_{\text {top }, 0}$ is therefore a measurement of the pressure of the vapor's amount initially present between the liquid's free surface and the piston.

When the electromagnet is switched-off, the pressure difference between both sides of the piston generates a force that accelerates the latter towards the liquid's surface. Note that the pressure above the piston is the atmospheric pressure, $P_{\text {atm }}$. When the piston finally impacts the liquid, rapid pressure changes are generated and used to promote fast bubble dynamic processes. 
The temporal evolution of the pressure under the piston, $P_{\mathrm{top}}$, and at the bottom of the reactor, $P_{\text {bott }}$, are both measured using two flush-mounted piezoelectric transducers $(601 \mathrm{H}$, Kistler) whose natural and cut-off frequencies are equal to 150 and $300 \mathrm{kHz}$ respectively. The pressure signals are amplified (5018A, Kistler) and recorded using a high-frequency oscilloscope (InfiniiVision MSO7054A, Agilent Technologies).

The pressure recordings obtained from the piezoelectric transducers contain information about both the background and the local pressures. The former, generated by the piston's impact, induces the nucleation and later the collapse of bubbles. As for the latter, it is generated by the bubbles' response. For that reason, along the manuscript, we compute an average effective chamber pressure using Newton's second law applied to the "piston" system. Neglecting the piston's weight and the friction forces, we express the average effective pressure below the piston, $\overline{P_{\text {int }}}$, equal to $P_{\text {top }}$, as:

$$
\overline{P_{\mathrm{int}}}=P_{\mathrm{top}}=P_{\mathrm{atm}}-\frac{m_{\mathrm{p}}}{S_{\mathrm{p}}} \frac{d V}{d t}
$$

where $V$ and $S_{\mathrm{p}}$ are respectively the piston's velocity and surface. In the following, all the peak-averaged pressures reported will be calculated from Eq.(1). In order to perform this calculation, we must have access to the temporal evolution of the piston's velocity which is obtained using a high-speed video camera (Phantom v711,Vision Research). The pressure signals and the image recordings are triggered by the output from one of the pressure transducers (threshold value $0.1 \mathrm{MPa}$ ). 
A schematic diagram of the apparatus is shown in Figure 1. When we study the behavior of pre-existing bubbles in the system during and after the piston's impact, we place the camera in position 2. For these high speed visualizations, we use a white LED lamp (RT220-20, Gardasoft Vision) for backlighting. As bubble nuclei naturally present in the system are difficult to observe before their activation, we resort to the generation of bubble nuclei using water electrolysis based on the following experimental protocol. A first electrode, acting as the cathode, is installed at the center of the lower piston (see Figure 1) whereas the second one, the anode, is placed on a lower side of the reactor. Both electrodes are connected to a pulsed electrical power source which is, in turn, connected to the computer. With this device, we can control the electrical signal that enables the electrolysis of water at a given instant before the piston's impact. In order to generate isolated bubble nuclei, we use short electric pulses with a frequency of the order of $1-10 \mathrm{kHz}$ and a voltage difference of the order of $25-50 \mathrm{~V}$. This procedure allows us to both generate and record hydrogen-filled bubbles saturated with vapor at the room temperature. The bubbles generated using this technique are of the order of $1-10 \mu \mathrm{m}$ at the cathode ${ }^{36}$.

During the expansion stage, these bubbles become large enough to be measured accurately using image processing techniques on the high-speed movie frames, which have a typical resolution of 4 microns/pixel. Bubble detection is carried out by applying a gray-level threshold to images once the background is removed with a rolling-ball algorithm. Subtracting the 
image background makes the area of the bubble images almost insensitive to the precise value of the threshold used. The bubble radii reported in this paper correspond to those of an equivalent circle with the same pixel area than the binary bubble image, $A$, that is, $R=\sqrt{A / \pi}$.

\section{Uncertainty measurements}

The instantaneous plate position obtained from the images were detected within an error of the order of the pixel size, which is of the order of few microns. The sampling acquisition rate was 50000 fps. Based on the piston's maximum velocities and impact heights presented herein, the plate velocity computed is estimated with an uncertainty lower than $1 \%$.

The data from the pressure transducers was acquired with an oscilloscope

at a sampling rate of $1.25 \mathrm{MS} \mathrm{s}^{-1}$. The voltage range was chosen as $\pm 8 \mathrm{~V}$ for all the runs of this study. The oscilloscope has an effective resolution of 8 bits at $1.25 \mathrm{MS} \mathrm{s}^{-1}$. This gives an output resolution of $62.5 \mathrm{kPa}$.

\section{The impact of a piston on a liquid enclosed in a fixed bottom chamber}

\section{Characteristic stages}

In this section we describe the characteristic stages of the process based on a qualitative description and through the analysis of the piston's position and velocity together with the pressure signal. Here we consider the simplest 
configuration, i.e. the fixed bottom configuration $(d=0)$.

Figure 2 presents a typical example of the temporal evolution of the piston's position, $H$, the piston's velocity, $V$, and the average pressure under the piston, $P_{\text {top }}$, as a function of time for an initial impact height of $H_{0}=$ $16 \mathrm{~mm}$. Time averaging is performed over five data samples. Every single sample is first shifted in time so that the origin $t=0$ is defined as the instant when the piston's velocity is maximum, $V_{\max }$.

Based on the analysis of Figure 2, we propose to divide the evolution of the system into three characteristic stages that are described below.

The acceleration stage (Figure 2, green) begins when the piston is released and ends when the velocity $V$ reaches its maximum value $V_{\max }$ at $t=t_{V_{\max }}$. During this stage, the pressure under the piston, $P_{\text {top }}$, is lower than the ambient pressure (i.e., $\left.P_{\text {atm }}\right)$ and the piston's acceleration, $a$, reaches a quasi-constant value as evidenced by the quasi-linear evolution of the piston's velocity $V$. The work of the external pressure force is mostly converted into the piston's kinetic energy, although during the very last instants of this stage, part of this energy is also used to increase the average temperature and pressure of the gas/vapor layer existing between the piston and the liquid's surface.

The compression stage (Figure 2, orange) is defined as the time evolution of the system between $t_{V_{\max }}$ and the moment when the chamber's pressure 
reaches its peak value, $P_{\text {top,max }}$. This instant is identified as the instant when the piston hits the liquid - gas interface. When the inner pressure forces equilibrate the outer pressure forces acting on the piston (which occurs at $\left.t_{V_{\max }}\right)$, the piston velocity starts decreasing, whilst the pressure of the gas/vapor layer keeps increasing. Figure 3 shows a typical example of the piston's position and velocity as well as the pressures measured with the piezoelectric transducers placed at the piston's surface and at the reactor's bottom together with the averaged pressure inferred from the piston's velocity data (Eq. 1). The first remark is that there is no observed lag between the two pressure signals $P_{\text {top }}$ and $P_{\text {bott }}$ (see Figure $3 \mathrm{~b}$ ), which can be explained by the fact that the characteristic liquid height, $H_{0,1}$ (Figure 1) is smaller than the wavelength of the wave induced during the impact, $\lambda=c_{1} / f_{\mathrm{i}}$, where $c_{1}$ is the speed of sound in the liquid and $f_{\mathrm{i}}$ the characteristic frequency generated during the impact. This results into a uniform background pressure inside the chamber. We also note that the effective pressure given by Eq. 1 is consistent with the direct pressure measurements of the transducers. In this case, the pressures are localized and it is possible to detect the high frequency fluctuations induced by the presence of surrounding bubbles. By using Eq. 1, local pressure fluctuations are smoothed out and we obtain a filtered evolution of the average effective pressure $\overline{P_{\text {int }}}$ acting on the piston.

The rebound stage(Figure 2, violet) is characterized by the minimum (negative) piston's velocity, $V_{\min }$. Once the piston impacts the liquid surface, the averaged pressure inside the chamber is much larger than the outer 
(atmospheric) pressure and the piston rebounds on the surface. During this stage, the pressure inside the chamber decreases and we will show that it is possible to observe tension states which induce the activation of bubble nuclei present in the system. After this stage, the chamber's pressure recovers back making existing bubbles implode.

Finally, successive impacts and rebounds of the piston occur with decreasing intensities due to the irreversible nature of the process.

In the next subsections, we quantify the generation of the pressure peaks and frequencies resulting from the piston's impact. As we will show below, these characteristic quantities are intrinsically related to the piston's maximum velocity and the thickness of the initially present gas/vapor layer above the liquid free surface.

\section{Peak pressures induced during the piston impact}

Figure 4 shows the computed chamber's maximum pressure, $P_{\text {top,max }}$ as a function of the piston's maximum velocity, $V_{\max }$, for various initial pressures $P_{\text {top }, 0}$. The peak pressures are obtained using Eq. (1) whereas the piston's maximum velocities are obtained from the digital processing of high-speed camera recordings. We observe that the amplitude of $P_{\text {top,max }}$ is proportional to the peak velocity $V_{\max }$. For the conditions tested here, pressures of the order of $0.5-3 \mathrm{MPa}$ are obtained. A relative good estimation of the peak velocity $V_{\max }$ obtained experimentally can result from Newton's second 
law considering that the friction forces are negligible and that the internal pressure is negligible compared to the atmospheric pressure during most of the acceleration stage,

$$
V_{\max }=\sqrt{\frac{2 P_{\mathrm{atm}} S_{\mathrm{p}} H_{0}}{m_{\mathrm{p}}}} .
$$

The experimental dependence of the piston's maximum velocity on the piston's initial position $H_{0}$ is presented in Figure 5

For reference, Figure 4 includes the maximum pressures expected if the gas/vapor layer was inexistent. In this idealized case, we can use the analytical solution derived in the context of the water-hammer theory, where a sudden pressure change is caused when a fluid in motion is suddenly forced to stop. This theory allows us to evaluate the maximum magnitude of the water hammer pulse from the Joukowsky equation as, $P_{\text {top, } \max }=\rho_{1} c_{1} V_{\max }$ where $\rho_{1}$ is the liquid's density. This theoretical solution is presented in Figure 4 for pure water $\left(\rho_{\mathrm{l}} c_{1}=1.490 \cdot 10^{6} \mathrm{~kg} \cdot \mathrm{m}^{-2} \cdot \mathrm{s}^{-1}\right.$, continuous black line). As we can see, the peak pressures and the slopes obtained from the experimental data are significantly smaller than the expected value for a sudden piston-water impact. This damping effect becomes more visible when the initial pressure under the piston $P_{\text {top }, 0}$ increases, thus revealing the importance of the presence of the gas/vapor layer during the last instants before the impact. In particular, the peak pressures obtained during the compression stage appear to be a function of the amount of vapor enclosed in the chamber. 
The influence of the gas/vapor layer on the peak pressures reached during the impact can be explained by the fact that during the instants before the impact (defined as the instant at which the piston's velocity is equal to zero), the compression of the gas/vapor layer tends to decrease the piston's velocity. The thickness of the gas/vapor layer at $t_{V_{\max }}$ referred to as $h_{\mathrm{c}}$, can be measured experimentally as the difference of the piston's position at the moment of maximum velocity and at the instant in which the piston's position reaches its minimal value. Figure 6 a presents the experimental values of $h_{\mathrm{c}}$ for various initial piston heights, $H_{0}$ and initial pressures, $P_{\mathrm{top}, 0}$. The values obtained for $h_{\mathrm{c}}$ range from 0.5 to $3.5 \mathrm{~mm}$. A simple model to predict $h_{\mathrm{c}}$ is proposed assuming that the gas/vapor layer is compressed during the acceleration stage following a polytropic law. From the initial time of fixed pressure $P_{\text {top }, 0}$ to the instant $t_{V_{\max }}$ when the inner pressure forces acting on the piston balance the atmospheric pressure forces, we assume that the pressure of the gas/vapor layer can be described by an effective polytropic evolution so that

$$
\frac{h_{\mathrm{c}}}{H_{0}}=\left(\frac{P_{\mathrm{top}, 0}}{P_{\mathrm{atm}}}\right)^{1 / \gamma_{\mathrm{eff}}}
$$

where $\gamma_{\text {eff }}$ is the effective polytropic coefficient of the gas/vapor mixture such that $P V^{\gamma_{\text {eff }}}=C$, where $C$ is a constant. The value of $\gamma_{\text {eff }}$ ranges from 0 for pure vapor substances if the compression is sufficiently slow $\left(P=P_{\text {sat }}\right)$ to $\gamma$, the gas/vapor polytropic coefficient, when the compression is very fast and the process is adiabatic ${ }^{?}$. The former case takes place when the compression is isothermal and the vapor condensation time scale is sufficiently 
short to maintain the vapor pressure at every instant.

The evolution of the dimensionless gas/vapor layer thickness $h_{\mathrm{c}} / H_{0}$ with respect to the dimensionless pressure $P_{\mathrm{top}, 0} / P_{\mathrm{atm}}$ is then tested against the polytropic relation given by Eq.(3) as presented in Figure 6b. For comparison, the isothermal (dashed line) and adiabatic (continuous line) transformations relative to the experimental conditions discussed here are superimposed to the experimental data points. Globally, the compression of the gas/vapor mixture in the acceleration stage can be modelized as an adiabatic process of an ideal gas, the agreement being remarkably good with the experimental data when $H_{0}$ is small.

We can make an analogy between the response of the rapidly compressed gas/vapor layer trapped between the piston and the liquid volume and the response of gas/vapor bubbles against pressure changes in diluted bubbly liquids ${ }^{?}$. The behavior of the gas/vapor layer can be discussed as a function of the Peclet and Sherwood numbers defined as the ratio between the averaged compression velocity $V_{\max }$ and the characteristic velocity of thermal diffusion $D^{\mathrm{T}} / H_{0}$ (to define the Peclet number) or mass diffusion $D^{\mathrm{M}} / H_{0}$ (to define the Sherwood number), where $D^{\mathrm{T}}$ and $D^{\mathrm{M}}$ are the averaged thermal and mass diffusivities of the gas/vapor mixture. For low Peclet numbers, the response of the system is isothermal and therefore the limiting response for a noncondensable gas $(P V=C)$ is different from the response of a condensable vapor (where $P=P_{\text {vap }}$ ). However, at large Peclet/Sherwood numbers, there is no time to exchange neither mass nor heat with the environment 
and we expect to find an adiabatic response of the gas/vapor mixture irrespective of the vapor content. In the present study, typical values of the Peclet numbers during the compression of the gas/vapor layer are of the order of $10^{3}-10^{4}$ which, according to the model presented in ${ }^{?}$, implies that the effective polytropic coefficient of the gas/vapor mixture corresponds to that of an adiabatic compression even when the vapor content is large. That is, the compression velocity during the acceleration stage is larger than the characteristic heat diffusion velocity in the gas/vapor layer.

The situation is slightly different during the compression stage. In this case the instantaneous Peclet and Sherwood numbers, proportional to the instantaneous compression velocity and the thickness of the gas/vapor layer, become small and therefore the compression is no longer adiabatic. Instead, the evolution of the gas/vapor pressure is influenced by mass and heat diffusion processes. Due to the pressure increase, vapor condenses slowing down the gas/vapor pressure increase and keeping the compression velocities high. This makes the reachable pressure peaks at the moment of impact dependent on the vapor content. As the vapor content increases (lower initial pressures), condensation processes are more important and the pressures generated at the impact are larger (see Figure 4).

Now that the characteristic length and velocity of the compression stage have been obtained, we focus on the analysis of the characteristic frequencies generated during the impact. 


\section{Characteristic frequencies induced during the piston's impact}

Along with the amplitude of the pressure wave, it is also important to characterize the frequencies generated during the impact. The pulses resulting from the impact generate a time-varying signal which requires the use of tools able to capture the appearance of a wide range of frequencies as a function of time. The choice of wavelets is then preferred compared to simple Fourier Transform analyses given that it allows us to quantify the intensity of various frequencies as a function of time.

In this work we show the result obtained from the treatment of a raw pressure signal obtained for an impact height $H_{0}=16 \mathrm{~mm}$ (Figure $7 \mathrm{a}$ ) using the Daubechies wavelet (Figure $7 \mathrm{p}$ ). We can see from Figure $7 \mathrm{~b}$ that the frequency content is rich during the impact when a pressure peak is detected. In this case, the highest values of the wavelet coefficients are found to lay in the range of $1-10 \mathrm{kHz}$.

In order to explain the characteristic frequency generated during the impact, we assume that the pressure increase is related to the compression time of the amount of gas/vapor trapped between the piston and the liquid free surface during the compression stage. The characteristic time of this stage, $t_{\mathrm{i}}$, can be obtained as the time difference between $t_{V_{\max }}$ and the instant when $P_{\text {top }}=P_{\text {top, max }}$. Note that this time defines a characteristic frequency related to the pressure variation induced by the compression of

the gas/vapor layer as $f_{\mathrm{i}}=t_{\mathrm{i}}^{-1}$. Figure 8 a presents the experimental values 
of $t_{\mathrm{i}}$ with respect to the impact height $H_{0}$ for various initial pressures $P_{\mathrm{top}, 0}$ below the piston. Typical values of the impact time are of the order of $0.2-$ $1.6 \mathrm{~ms}$. Consistent with the results obtained from the wavelet analysis, the impact frequencies $f_{\mathrm{i}}$ are found to range between approximately $1 \mathrm{kHz}$ for the largest value of $P_{\text {top }, 0}$ tested and approximately $3 \mathrm{kHz}$ for the smallest value of $P_{\text {top }, 0}$ (see Figure 9a). The amount of gas/vapor trapped between the piston and the liquid's free surface influences indeed the time lapse over which compression occurs. On the contrary, a clear dependence of $t_{\mathrm{i}}$ on $H_{0}$ is not observed (except maybe for the largest values of $\left.P_{\mathrm{top}, 0}\right)$. This result stresses out the role of the gas/vapor layer where the amount of vapor controls not only the peak pressure obtained at the piston's impact but also the characteristic frequency of the pressure variation induced in the liquid medium.

Defining a characteristic compression time based on the characteristic length of the gas layer and peak velocity of the process, $t_{\mathrm{c}}=h_{\mathrm{c}} / V_{\max }$, a nondimensional time and its corresponding nondimensional frequency can be obtained. These nondimensional quantities are expected to depend only on the initial non-dimensional pressures, $\frac{P_{\mathrm{top}, 0}}{P_{\mathrm{atm}}}$ and the effective polytropic coefficient, $\gamma_{\text {eff }}$, i.e. $\frac{t_{\mathrm{c}} V_{\max }}{H_{0}}=\left(\frac{P_{\mathrm{top}, 0}}{P_{\mathrm{atm}}}\right)^{\frac{1}{\gamma_{\mathrm{eff}}}}$ and $\frac{f_{\mathrm{c}} H_{0}}{V_{\max }}=\left(\frac{P_{\mathrm{top}, 0}}{P_{\mathrm{atm}}}\right)^{-\frac{1}{\gamma_{\mathrm{eff}}}}$ respectively. Figures $8 \mathrm{~b}$ and $9 \mathrm{~b}$ depict the nondimensional times and frequencies obtained as a function of the dimensionless pressures $P_{\text {top }, 0} / P_{\text {atm }}$ and for various impact heights $H_{0}$. Along with the experimental data, two characteristic evolutions based on the tested initial pressures $P_{\text {top }, 0}$ are represented in these figures for comparison, i.e. an isothermal (dashed line) and an adi- 
abatic (continuous line) compression. Again, the characteristic time during the impact increases when increasing the initial pressures $P_{\text {top }, 0}$, revealing the importance of the amount of gas/vapor trapped in the small layer between the piston and the liquid's surface. The collapse of the data points on the adiabatic trend is relatively good confirming that the effective polytropic coefficient approaches the adiabatic limit expected in the compression stage as well.

As a summary of the previous discussion, we conclude from this part of the analysis that the impact of the piston on the liquid's surface generates an approximately spatially uniform variation of the pressure in the system. Both the characteristic amplitude and frequency of the excitation are strongly influenced by the dynamics of the gas/vapor layer trapped between the piston and the liquid's surface. In addition to this parameter, the amplitude of the pressure variation induced in the system strongly depends on the impact velocity and the medium's compressibility. The characteristic frequency generated by the impact is also related to the maximum velocity.

Using Equation (2) to determine the equations of the pressure peak and the characteristic frequency as a function of controllable variables we obtain:

$$
\begin{aligned}
P_{\max } & =C_{0} \rho_{\mathrm{l}} c_{1} \sqrt{\frac{2 P_{\mathrm{atm}} S_{\mathrm{p}} H_{0}}{m_{\mathrm{p}}}} \\
f_{\mathrm{i}} & =\sqrt{\frac{2 P_{\mathrm{atm}} S_{\mathrm{p}}}{H_{0} m_{\mathrm{p}}}}\left(\frac{P_{\mathrm{atm}}}{P_{\mathrm{top}, 0}}\right)^{\frac{1}{\gamma}}
\end{aligned}
$$


where $C_{0}$ is a constant that includes the damping effect due to the presence of gas. It is experimentally found to be between $1 / 3$ and $1 / 2$.

It is interesting to note that we expect to generate higher and faster pressure changes and frequencies when increasing the reference pressure $P_{\text {top }, 0}$

and decreasing the piston's mass per unit surface $m_{\mathrm{p}} / S_{\mathrm{p}}$ (which is a measure of the inertia per unit surface). On the contrary, the effect of the impact height $H_{0}$ is more intricate. In fact, if we increase $H_{0}$, the intensity of the impact could be augmented at the expense of decreasing the characteristic frequencies generated during the impact. As we will see in the next section, this is going to restrict the range of bubble sizes that undergo an intense collapse.

\section{Bubble dynamics effects}

In the previous section, we have determined both the maximum pressures generated in the reactor and the characteristic frequencies resulting from the piston's impact. Now we focus our attention on the behavior of pre-existing bubbles in the system during and after the piston's impact. The bubble's radius is obtained from the analysis of the images obtained from the highspeed camera recordings. Figure 10 illustrates an example of the bubble's contour for the bubble evolution resulting from the pressure change induced by the piston impact shown in Figure 11 . 
The collapse of a bubble after a sudden increase of the external pressure is theoretically identified with the so-called Rayleigh collapse. Given a pressure jump between the bubble internal and the external pressure, $\Delta P=P_{\max }-P_{\text {top }, 0} \approx P_{\max }$, the time lag between the instants when the radius is maximum, $R_{\max }$ and minimum, $R_{\min }$ is given by the Rayleigh collapse time $\left(t_{\text {Rayleigh }}\right)$. The analytical expression for $t_{\text {Rayleigh }}$ was derived by Rayleigh in $1917^{37}$,

$$
t_{\text {Rayleigh }}=0.915\left(\frac{\rho_{1} R_{\max }^{2}}{P_{\max }}\right)^{1 / 2} .
$$

The response of the bubbles is shown to be different depending on the ratio between the characteristic time generated during the impact, $t_{\mathrm{i}}=$ $f_{\mathrm{i}}^{-1}$ (where $f_{\mathrm{i}}$ is theoretically obtained using Equation 5), and the bubble's characteristic time $t_{\text {Rayleigh. }}$. Thus, we define a critical bubble radius $R_{\mathrm{c}}$ as the radius for which $t_{\mathrm{i}}=t_{\text {Rayleigh }}$, so that

$$
R_{\mathrm{c}}=\frac{H_{0}}{1.884}\left(\frac{c_{1}^{2} m_{\mathrm{p}}}{H_{0} S_{\mathrm{p}} P_{\mathrm{atm}}}\right)^{1 / 4}\left(\frac{P_{\mathrm{top}, 0}}{P_{\mathrm{atm}}}\right)^{1 / \gamma}
$$

Typical values of the critical radius $R_{\mathrm{c}}$ are found to be of the order of $1 \mathrm{~mm}$ for the conditions reported in this manuscript.

The experimental set-up allows us to control the size of bubbles before the impact. To investigate the response of bubbles with an initial size below $R_{\mathrm{c}}$, we use the technique described in section Experimental setup and measurements based on electrolysis. On the other hand, in order to explore 
the behavior of bubbles with a characteristic size larger than the critical radius $R_{\mathrm{c}}$, we have performed additional experiments in which we managed to trap inside the liquid large gas/vapor bags generated during the initial depressurization process. These gas/vapor bags are not spherical, but one can still obtain the evolution of the characteristic size of the system from the measurement of the projected area obtained from the images, so that $S_{\text {bubble }}=\pi R_{\text {bub }}^{2}$

Figure 11 presents both the temporal evolution of the pressure under the piston, $P_{\text {top }}$ and the temporal evolution of the bubble's size, $R_{\text {bub }}$, obtained in two different experiments. Figure 11 a depicts the evolution of a bubble of the order of $R_{\mathrm{bub}} \approx R_{\mathrm{c}}$ and Figure $11 \mathrm{~b}$ shows the evolution of an initial bubble nuclei of the order of few microns $\left(R_{\text {bub }} \ll R_{\mathrm{c}}\right)$. In both cases, the bubble's compression begins before the piston's impact, which confirms the increase of the system's pressure due to the gas/vapor compression described in the preceding section. We can clearly see that the dynamic response of the bubbles is remarkably different depending on their radii. When the initial bubble radius is of the order of the critical radius $R_{\mathrm{c}}$ (Equation 7 ) a violent bubble collapse takes place during the first pressure peak generated by the piston impact. As the evolution of $R_{\text {bub }}$ shows, the compression of the bubble at its final stages (between 0 and $0.2 \mathrm{~ms}$ ) is extremely fast due to the large pressure difference generated between the bubble's interior and the background pressure generated during the impact. This is a consequence of the comparable characteristic response time of the bubble and the characteristic impact time which creates a pressure difference between the inside 
of the bubble and the background system pressure that finally leads to a strong bubble collapse during the compression stage.

Bubbles much smaller than $R_{\mathrm{c}}$ (e.g. micron-sized bubbles) slowly compress during the initial compression stage due to the small characteristic compression time with respect to the fast bubble's response time (inversely proportional to the bubble's resonance frequency). Thus, bubbles with radii smaller than $R_{\mathrm{c}}$ follow the pressure's increase generated during the compression stage without undergoing any violent collapse during the initial compression stage. During the piston's rebound stage, negative pressures are generated in the liquid which correspond to tension states where small bubble nuclei expand significantly. When a tension state occurs (see Figure 11.), the small bubbles are unable to adjust their pressure to the background pressure and their sizes increase by at least one order of magnitude reaching their maximum values. The bubbles finally collapse when the system pressure is recovered undergoing a series of rebounds until the bubble pressure re-equilibrates with the surrounding pressure. Since the intensity of the waves decays in time, the first expansion-collapse is the most violent.

Choosing the maximum radius, $R_{\max }$, and the collapse time, $t_{\mathrm{col}}$ as characteristic variables of the process, we plot the temporal evolution of the radius of different bubbles (Figure $12 \mathrm{a}$ ). The results are shown in dimensionless form in Figure 12 b. As we can see, bubbles having individually different behaviors have the same normalized response as predicted by the Rayleigh collapse theory. 
The collapse of bubbles induce an important agitation at very small scales due to the formation of a liquid jet during the last instants of their collapse and a significant acceleration in their translational motion. Figures 13. 14 and 15 show characteristic snapshots of the jet formation process during the bubble collapse. This is interesting given that the bubbles act as a small source of mixing during the last instants of the collapse, where the characteristic scales are below $1 \mathrm{~mm}$. 
Jet formation results from asymmetries during the collapse induced by pressure differences across the cavity. The jet velocities calculated based on Figures 13, 14 and 15 are respectively $0.7653,0.5952$ and $1.607 \mathrm{~m} \mathrm{~s}^{-1}$. Some previous studies in the literature have investigated the effect of newtonian and non-newtonian liquids on the jet formation ${ }^{38 / 39}$, showing that jets have an impact on the instability patterns of cylindrical jets 40 . Brown and Williams ${ }^{39}$ have studied the effect of the addition of the polyacrylamide (PAA) to a newtonian liquid made of syrup and water. The liquid jet velocities reported for the newtonian fluid varied from 4 to $6 \mathrm{~ms}^{-1}$. Upon the addition of $100 \mathrm{ppm}$ of PAA, the jet velocities were 1.5 times smaller. We can then conclude that despite the low impact velocities used in this work (of the order of $5 \mathrm{~m} \mathrm{~s}^{-1}$ ), the current technique is able to generate jets during the collapse of bubbles with velocities that are comparable in orders

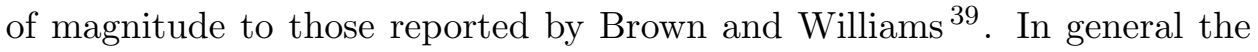
presence of a source of asymmetry, as for example solid walls or the presence of surrounding bubbles, may also significantly modify the maximum jet velocities reached during the impact 41 .

In addition to the jet velocity, it is also possible to characterize the intensity of the mixing process measuring the temporal evolution of the translational velocity of various bubbles (Figure 16). We can observe that every bubble has an approximately constant velocity before reaching its maximum radius, $R_{\max }$. This velocity is assumed to be the flow velocity, $V_{\text {flow }}$, induced by both the piston impact and the subsequent pressure wave traveling within the liquid. In order to obtain the translation velocity attributed 
to the bubble collapse process, we can subtract the flow velocity, $V_{\text {flow }}$ from the bubble's total velocity $V_{\text {bub }}$. Figure 16 also presents the dimensionless plots of $\frac{\left(V_{\mathrm{bub}}-V_{\mathrm{flow}}\right) t_{\mathrm{col}}}{R_{\max }}$ as a function of the dimensionless radius, $\frac{R}{R_{\max }}$. We differentiate two stages during the collapse: the early compression and the final collapse. The early compression takes place when the bubble starts to compress and increases its velocity at a constant rate. During the final stage of the collapse, the bubble is about to reach its minimum radius, $R_{\min }$, and the acceleration increases the translational velocity by about one order of magnitude when a jet generated during the bubble collapse (see Figure 13). It is interesting to note that in these cases the nondimensional translational velocity can be as large as two times the characteristic compression veloc-

ity $R_{\max } / t_{\mathrm{col}}$. This shows the potential of this technique to induce strong agitation at very small scales (of the order of the jet generated during the bubble collapse).

\section{Discussion and conclusions}

This work describes the dynamics of bubbles present in liquids after the impact of a piston on the liquid free surface. Using high-speed camera visualizations and pressure measurements, three characteristic stages of the process have been identified : a) the acceleration stage defined as the time lag over which the piston is accelerated (pressure inside the chamber is lower than the reference pressure), b) the compression stage represented by the time lag between $t_{V_{\max }}$ (end of the acceleration stage) and the instant when 
the pressure surge reaches its maximum value and c) the rebound stage characterized by a rebound of the piston after its impact on the liquid free surface due to a large excess of pressure forces acting below the piston.

We identify two quantities of major importance to characterize the system response: the peak pressure reached during the impact and the characteristic frequency of the pressure transmitted to the liquid medium. The pressure peaks observed vary between 0.5 and $3 \mathrm{MPa}$ depending on the impact velocity and the amount of water vapor trapped in a small gas/vapor layer under the piston. Unlike techniques based on ultrasound, in the new device pressures are efficiently transmitted to the liquid medium without any significant attenuation. Indeed the pressure can be assumed to be almost uniform in the liquid volume. The characteristic impact frequencies transmitted to the liquid medium have been shown to range between 0.5 and $4.5 \mathrm{kHz}$. These frequencies are of the same order of magnitude as the ones required to excite nuclei present in a volume of water to generate large cavitation bubbles.

Both the peak pressures and the impact frequencies are shown to depend on the amount of gas/water vapor trapped between the piston and the liquid free surface. Based on some analogies with the compression of gas/vapor bubbles in a bubbly liquid, we present a simple model developed to correlate the effective internal pressure and the piston's maximum velocity based on a modified Joukowsky equation which accounts for the presence of the gas/vapor layer. 
The visualization of bubbles initially present in the medium showed that bubbles behave differently depending on their size. Based on the ratio between the characteristic compression time related to the compression of the gas/vapor layer below the piston and the Rayleigh collapse time of the bubble, we find a critical radius that depends on the impact velocity and both the initial and the reference pressures. During the initial pressure peak induced by the impact of the piston, bubbles smaller than this critical radius (of the order of millimeters in our conditions) tend to follow the system's pressure evolution and compress slowly, whereas bigger bubbles collapse violently. In the rebound stage, tension occurs and small bubbles expand and collapse violently at later times. In general, the collapse of bubbles present in the medium creates an important agitation at very small scales (of the order of microns) and induces the generation of pressure waves presumably generated by this collapse. The possibility to use this process to promote chemical and physical processes on complex systems containing two or more phases (solid/liquid/gas) is currently under investigation.

\section{Acknowledgment}

The authors would like to acknowledge the support of Francis Luck, Francois Montel and Philippe Guibert during different stages of this project. We also acknowledge the support of the Spanish Ministry of Economy and Competitiveness through grants DPI2014-59292-C3-1-P and DPI2015-71901-REDT, 
partly funded through European Funds.

\section{Literature Cited}

1. van Wijngaarden L. Mechanics of collapsing cavitation bubbles. Ultrasonics Sonochemistry . 2016;29:524-527.

2. Rayleigh L. VIII. on the pressure developed in a liquid during the collapse of a spherical cavity. Philosophical Magazine Series 6 . 1917;34(200):94-98.

3. Neppiras E. Macrosonics in industry 1. Introduction. Ultrasonics . 1972;10(1):9-13.

4. Moholkar VS, Warmoeskerken MMCG, Ohl CD, Prosperetti A. Mechanism of mass-transfer enhancement in textiles by ultrasound. AIChE Journal . 2004;50(1):58-64.

5. Mason TJ. Ultrasonic cleaning: An historical perspective. Ultrasonics Sonochemistry . 2016;29:519-523.

6. Dular M, Griessler-Bulc T, Gutierrez-Aguirre I, Heath E, Kosjek T, Klemeni AK, Oder M, Petkovek M, Raki N, Ravnikar M, arc A, irok B, Zupanc M, itnik M, Kompare B. Use of hydrodynamic cavitation in (waste)water treatment. Ultrasonics Sonochemistry . 2016;29:577-588.

7. Braeutigam P, Franke M, Schneider RJ, Lehmann A, Stolle A, Ondruschka B. Degradation of carbamazepine in environmentally relevant 
concentrations in water by Hydrodynamic-Acoustic-Cavitation (HAC). Water Research . 2012;46(7):2469-2477.

8. Gong C. Ultrasound induced cavitation and sonochemical effects. Ph.D. Thesis, MIT. 1999.

9. Gogate PR, Pandit AB. A review of imperative technologies for wastewater treatment I: oxidation technologies at ambient conditions. Advances in Environmental Research . 2004;8(3-4):501-551.

10. Hung HM, Hoffmann MR. Kinetics and mechanism of the sonolytic degradation of chlorinated hydrocarbons: Frequency effects. Journal of Physical Chemistry A . 1999;103(15):2734-2739.

11. Mason TJ. A Survey of Commercially Available Sources of Ultrasound Suitable for Sonochemistry. In: Sonochemistry - Uses of Ultrasounds in Chemistry. Cambridge UK: Royal Society of Chemistry. 1990:60-68.

12. Gogate PR, Pandit AB. A review and assessment of hydrodynamic cavitation as a technology for the future. Ultrasonics Sonochemistry . 2005;12(1-2):21-27. Ninth Conference of the European Society of Sonochemistry.

13. Gent AN, Lindley PB. Internal rupture of bonded rubber cylinders in tension . 1959;249(1257):195-205.

14. Ball JM. Discontinuous equilibrium solutions and cavitation in nonlinear elasticity. Philosophical Transactions of the Royal Society of London Series A, Mathematical and Physical Sciences . 1982;306(1496):557-611. 
15. Meyers MA. Dynamic Behavior of Materials. Wiley. 1994.

16. Hopkinson B. A method of measuring the pressure produced in the detonation of high explosives or by the impact of bullets. Philosophical Transactions of the Royal Society of London Series A, Containing Papers of a Mathematical or Physical Character . 1914;213(497-508):437456.

17. Blake Jr F. Onset of cavitation in liquids. Ph.D. Thesis, Harvard University. 1949.

18. Mørch KA. Cavitation nuclei and bubble formationa dynamic liquid-solid interface problem. Journal of Fluids Engineering . 2000;122(3):494-498.

19. Ando K, Liu AQ, Ohl CD. Homogeneous nucleation in water in microfluidic channels. Physical Review Letters . 2012;109:044501.

20. Quinto-Su PA, Ando K. Nucleating bubble clouds with a pair of laserinduced shocks and bubbles. Journal of Fluid Mechanics . 2013;733.

21. Fuster D, Pham K, Zaleski S. Stability of bubbly liquids and its connection to the process of cavitation inception. Physics of Fluids . 2014;26(4):042002.

22. Andersen A, Mørch KA. Cavitation nuclei in water exposed to transient pressures. Journal of Fluid Mechanics . 2015;771:424-448.

23. Fourest T, Laurens JM, Deletombe E, Dupas J, Arrigoni M. Analysis of bubbles dynamics created by hydrodynamic ram in confined geometries 
using the rayleigh-plesset equation. International Journal of Impact Engineering . 2014;73:66-74.

24. Dear J, Field J. A study of the collapse of arrays of cavities. Journal of Fluid Mechanics . 1988;190:409-425.

25. Chesterman W. The dynamics of small transient cavities. Proceedings of the Physical Society, Section B . 1952;65(11):846.

26. Field J, Lesser M, Dear J. Studies of two-dimensional liquid-wedge impact and their relevance to liquid-drop impact problems. Proceedings of the Royal Society of London A: Mathematical, Physical and Engineering Sciences . 1985;401(1821):225-249. .

27. Lesser M, Field J. The impact of compressible liquids. Annual review of fluid mechanics . 1983;15(1):97-122.

28. Daily J, Pendlebury J, Langley K, Hurd R, Thomson S, Truscott T. Catastrophic cracking courtesy of quiescent cavitation. Physics of Fluids . 2014;26(9):091107.

29. Rodríguez-Rodríguez J, Casado-Chacón A, Fuster D. Physics of beer tapping. Physical Review Letters . 2014;113:214501.

30. Dular M, Coutier-Delgosha O. Thermodynamic effects during growth and collapse of a single cavitation bubble. Journal of Fluid Mechanics . 2013;736:44-66.

31. Fuster D, Dutilleul H, Guibert P, Da Costa P, Zaleski S. Dispositif et 
procede de traitement de liquide par cavitation. 2015. SATT Lutech, FR 1558892.

32. Flint EB, Suslick KS. The temperature of cavitation. Science . 1991;253(5026):1397-1399.

33. Hauke G, Fuster D, Dopazo C. Dynamics of a single cavitating and reacting bubble. Physical Review E . 2007;75(066310):1-14.

34. Fuster D, Montel F. Mass transfer effects on linear wave propagation in diluted bubbly liquids. Journal of Fluid Mechanics . 2015;779:598-621.

35. Rivas DF, Stricker L, Zijlstra AG, Gardeniers HJ, Lohse D, Prosperetti A. Ultrasound artificially nucleated bubbles and their sonochemical radical production. Ultrasonics Sonochemistry . 2013;20(1):510-524.

36. Medina A. Experimental and analytical study of the interaction between short acoustic pulses and small clouds of microbubbles. $\mathrm{PhD}$ Thesis, Universidad Carlos III. 2015.

37. Brennen CE. Cavitation and bubble dynamics. Oxford engineering science series 44, Oxford University Press. 1995.

38. Williams P, Williams P, Brown S. A study of liquid jets formed by bubble collapse under shock waves in elastic and newtonian liquids. Journal of Non-Newtonian Fluid Mechanics . 1998;76(1-3):307-325.

39. Brown S, Williams P. The tensile behaviour of elastic liquids under dynamic stressing. Journal of Non-Newtonian Fluid Mechanics . 2000;90(1):1 - 11. 
40. Gordon M, Yerushalmi J, Shinnar R. Instability of jets of nonnewtonian fluids. Transactions of the Society of Rheology . 1973;17(2):303-324. .

41. Obreschkow D, Tinguely M, Dorsaz N, Kobel P, De Bosset A, Farhat M. Universal scaling law for jets of collapsing bubbles. Physical Review Letters . 2011;107(20):204501. 


\section{List of Figures}

$1 \quad$ Sketch of the experimental apparatus . . . . . . . . . . . 38

$2 \quad$ Characteristic stages of the process . . . . . . . . . . . . 39

$3 \quad$ Example of the evolution of the position, velocity and pressures 40

4 Figure: Maximum pressure under the piston as a function of the maximum velocity. Table: Theoretical solution to the

water-hammer theory and the experimental linear fits of the maximum pressure as a function of the maximum velocity. . . 41

5 Temporal evolution of the piston's velocity for various impact heights. . . . . . . . . . . . . . . . . . 42

$6 \quad$ Experimental gas/vapor layer thickness as a function of the impact height and the nondimensional gas/vapor layer thickness as a function of the dimensionless pressure. . . . . . . . . 43

$7 \quad$ Experimental pressure signal and its Daubechies wavelet . . . 44

$8 \quad$ Impact time as a function of the impact height and the nondimensional impact time as a function of the dimensionless pressures. . . . . . . . . . . . . . . . . 4 45

$9 \quad$ Impact frequency as a function of the impact height and the nondimensional impact frequency as a function of the dimensionless pressures. . . . . . . . . . . . . . . . . . 46

10 Example of the contour-capturing algorithm applied to the high-speed video frames during the bubble expansion . . . . . 47

11 Evolution of the radii of a big and a small bubble with the experimental pressures. . . . . . . . . . . . . . . . . 48 
12 Temporal evolution of the radii of various bubbles and their dimensionless plot. . . . . . . . . . . . . . . . 49

13 Example 1: Snapshots during the collapse of a single bubble generated by electrolysis. . . . . . . . . . . . . 50

14 Example 2: Snapshots during the collapse of a single bubble generated by electrolysis. . . . . . . . . . . . . . 50

15 Example 3: Snapshots during the collapse of a single bubble generated by electrolysis. . . . . . . . . . . . . . 51

16 Temporal evolution of the translational velocity of various bubbles and its nondimensional evolution. . . . . . . . . . 52

\section{List of Tables}

$1 \quad$ Figure: Maximum pressure under the piston as a function of the maximum velocity. Table: Theoretical solution to the water-hammer theory and the experimental linear fits of the maximum pressure as a function of the maximum velocity. . 41 
List of Figures 


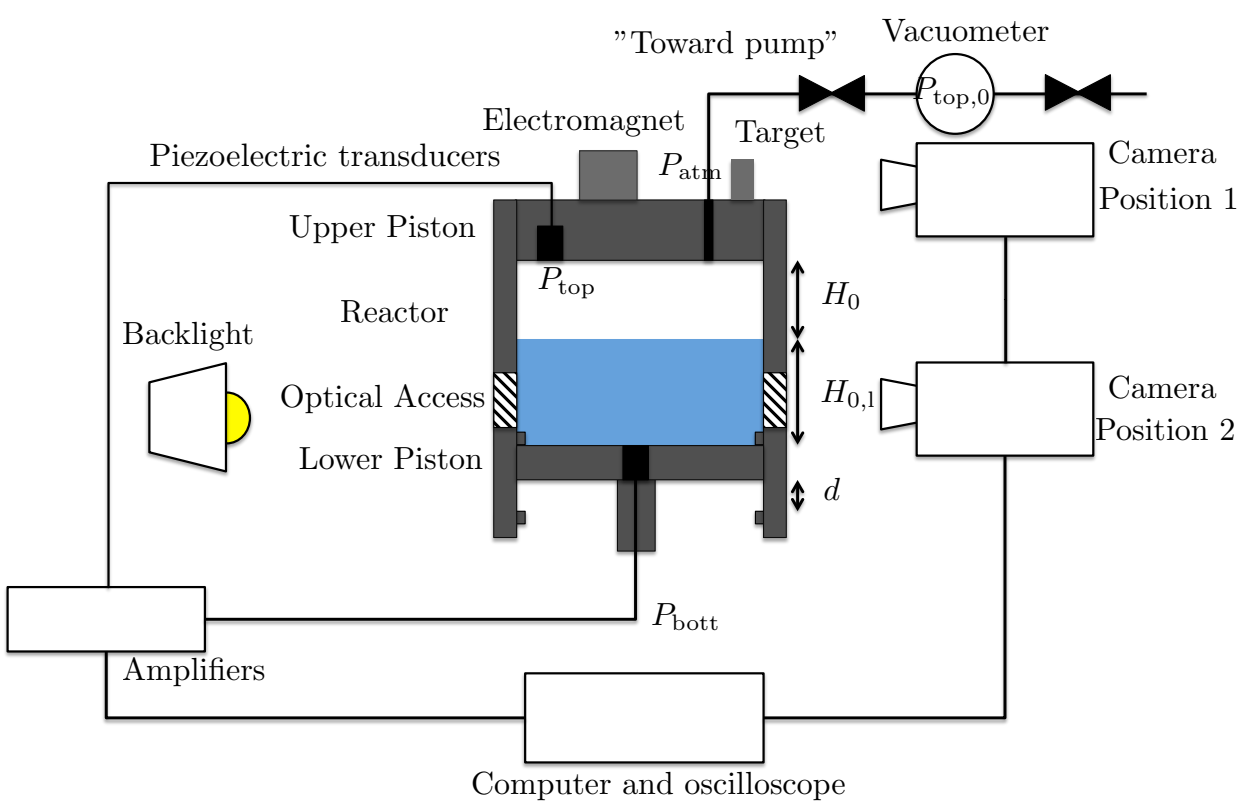

Figure 1: Sketch of the experimental apparatus used to induce cavitation with a piston and to observe the bubble dynamic response. 

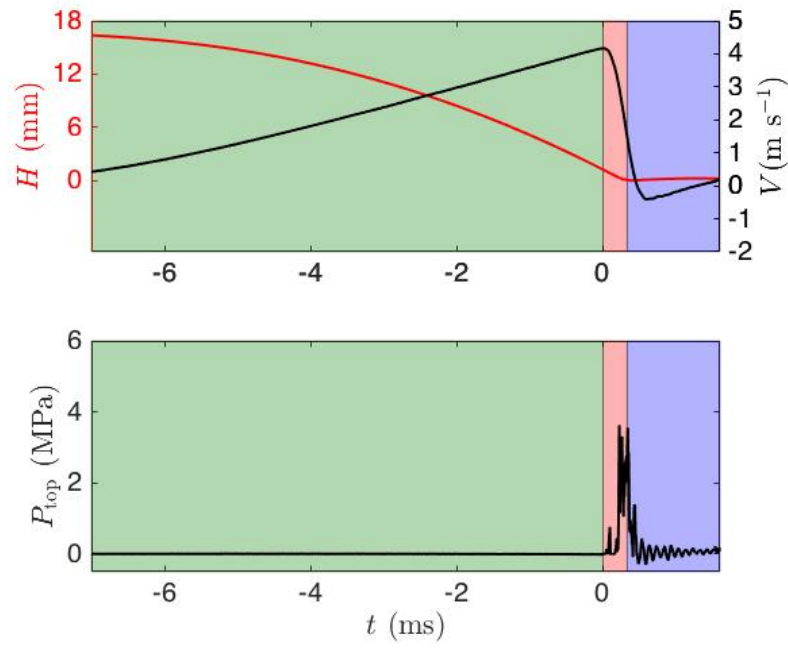

Figure 2: Top: Temporal evolution of the average position (red line) and piston velocity (black line). Bottom: Pressure under the piston for $H_{0}=16 \mathrm{~mm}$. 


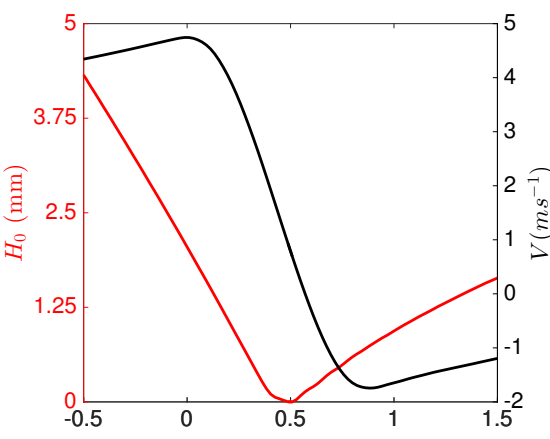

(a)

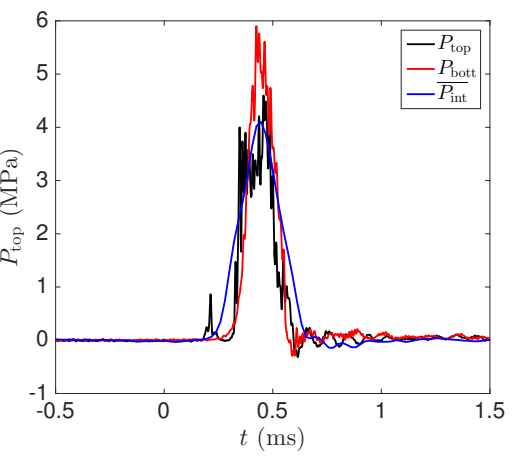

(b)

Figure 3: Left: Temporal evolution of the position (red line) and the piston velocity (black line). Right: Temporal evolution of the pressures experimentally measured $\left(P_{\mathrm{top}}\right.$ and $\left.P_{\mathrm{bott}}\right)$ and the effective pressure below the piston $\left(\overline{P_{\mathrm{int}}}\right)$. 

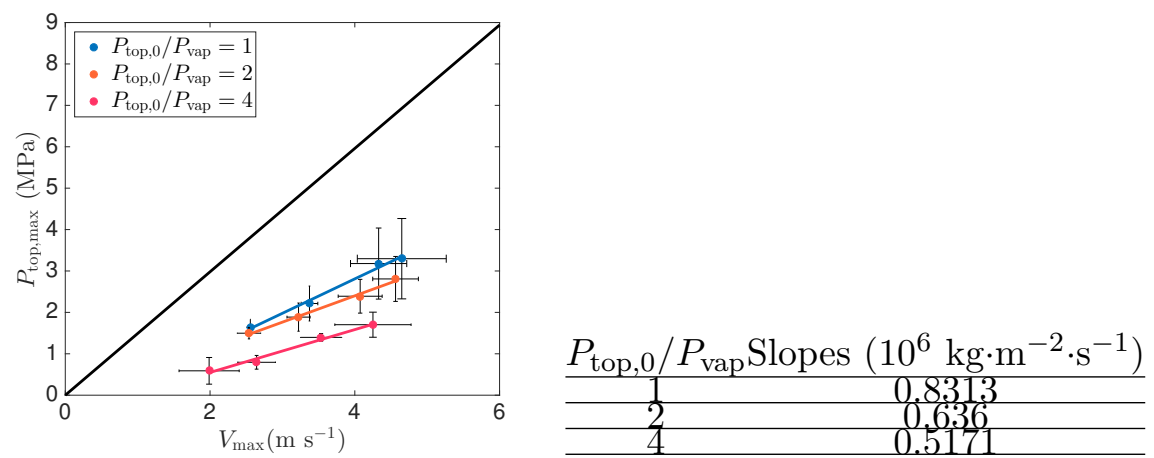

Figure $4 \&$ Table 1: Maximum pressure under the piston $P_{\text {top, max }}$ as a function of the maximum velocity $V_{\max }$ for various initial pressures $P_{\text {top }, 0} / P_{\text {vap }}$. Theoretical solution to the water-hammer theory (black), $P_{\text {top }, 0} / P_{\text {vap }}=1$ (blue), $P_{\text {top }, 0} / P_{\text {vap }}=$ 2 (orange), $P_{\text {top }, 0} / P_{\text {vap }}=4$ (pink). In the table we show the experimental linear fits of the maximum pressure $P_{\mathrm{top}, \max }$ as a function of the maximum velocity $V_{\max }$ for various initial pressures $P_{\mathrm{top}, 0} / P_{\mathrm{vap}}$. 


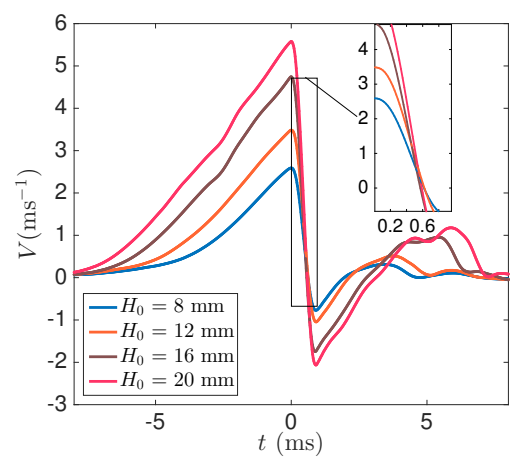

Figure 5: Temporal evolution of the piston's velocity $V$ for various impact heights $H_{0}$. 


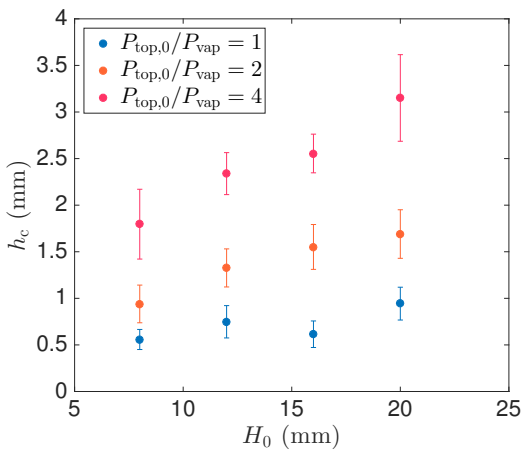

(a)

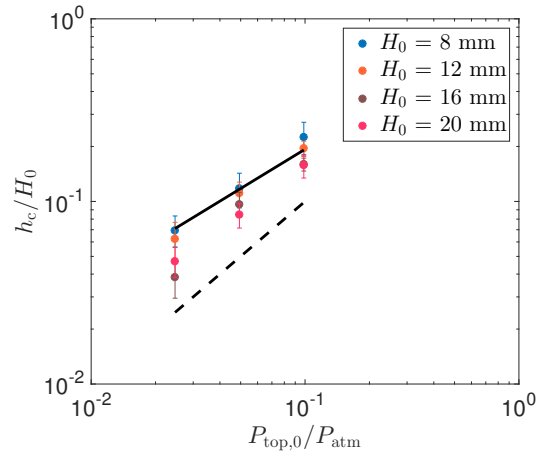

(b)

Figure 6: Left: Experimental gas/vapor layer thickness $h_{\mathrm{c}}$ at $t_{V_{\max }}$ as a function of the impact height $H_{0}$ for different initial pressure under the piston $P_{\mathrm{top}, 0}$. Right: Non-dimensional gas/vapor layer thickness as a function of the dimensionless pressure $P_{\mathrm{top}, 0} / P_{\mathrm{atm}}$. Experimental data (o), adiabatic transformation (-) and isothermal transformation $(--)$. 


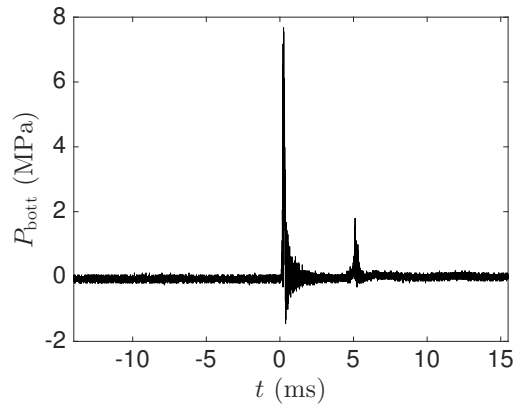

(a)

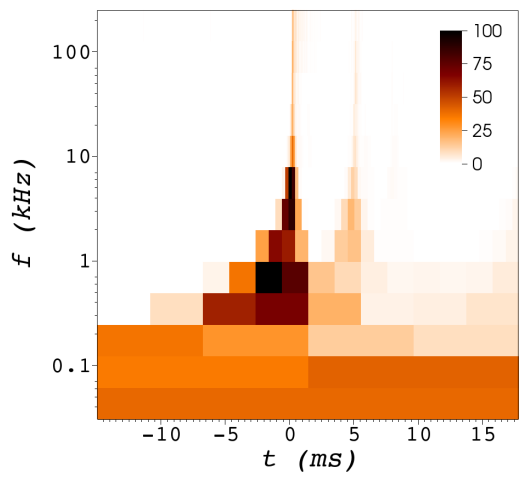

(b)

Figure 7: Left: Pressure signal at the bottom of the reactor obtained for $H_{0}=$ $16 \mathrm{~mm}$. Right: Daubechies wavelet of the pressure signal presented in (a). The color bar represents the absolute values of the wavelet coefficients, which represent a measure of the signal intensity. 


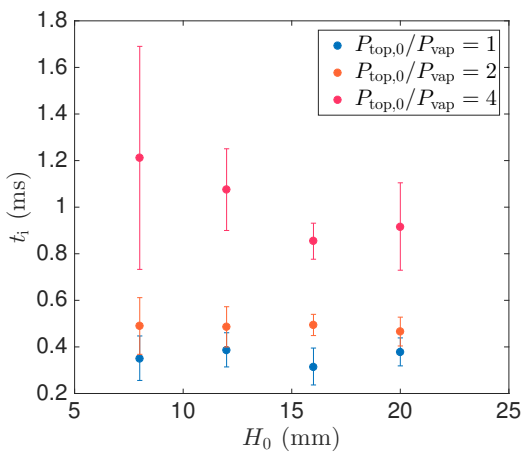

(a)

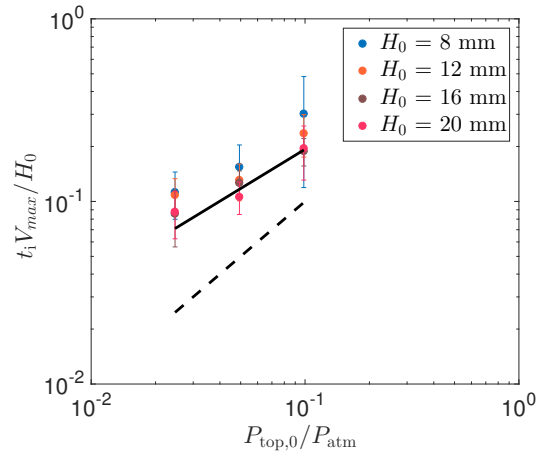

(b)

Figure 8: Left: Impact time $t_{\mathrm{i}}$ as a function of the impact height $H_{0}$. Right: Nondimensional impact time $t_{\mathrm{c}} V_{\max } / H_{0}$ as a function of the dimensionless pressures $P_{\text {top }, 0} / P_{\text {atm }}$. Experimental data (o), adiabatic transformation $(-)$ and isothermal transformation $(--)$. 


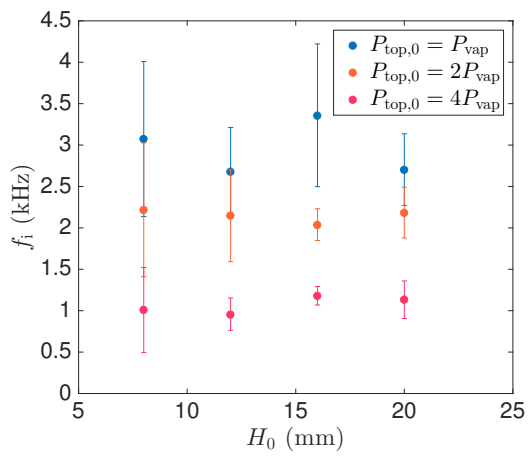

(a)

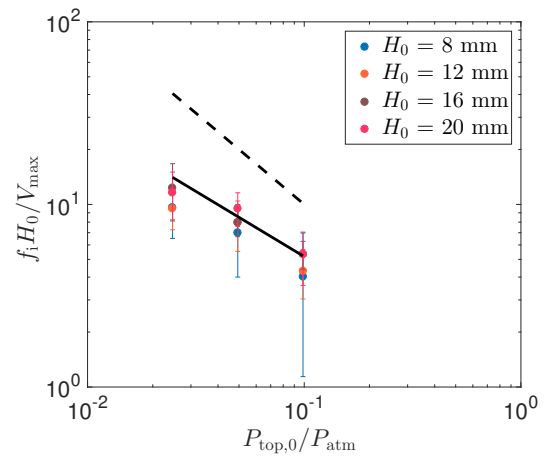

(b)

Figure 9: Left: Impact frequency $f_{\mathrm{i}}$ as a function of the impact height $H_{0}$. Right: Non-dimensional frequency $f_{\mathrm{c}} H_{0} / V_{\max }$ as a function of the dimensionless pressure $P_{\text {top }, 0} / P_{\text {atm }}$. Experimental data (o), adiabatic transformation $(-)$ and isothermal transformation $(--)$. 

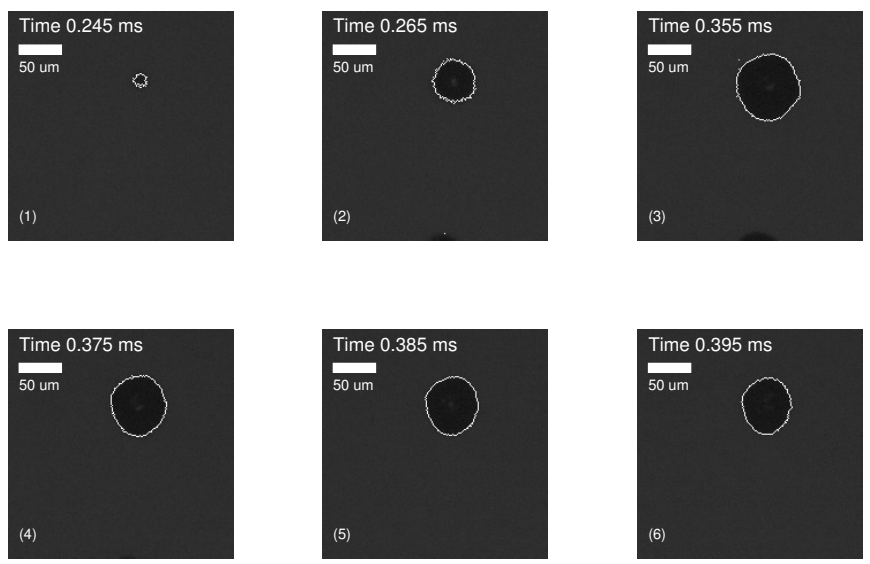

Figure 10: Example of the contour-capturing algorithm applied to the high-speed video frames during the bubble expansion plotted in Figure 11p. Frame rate: 50 000 fps. 


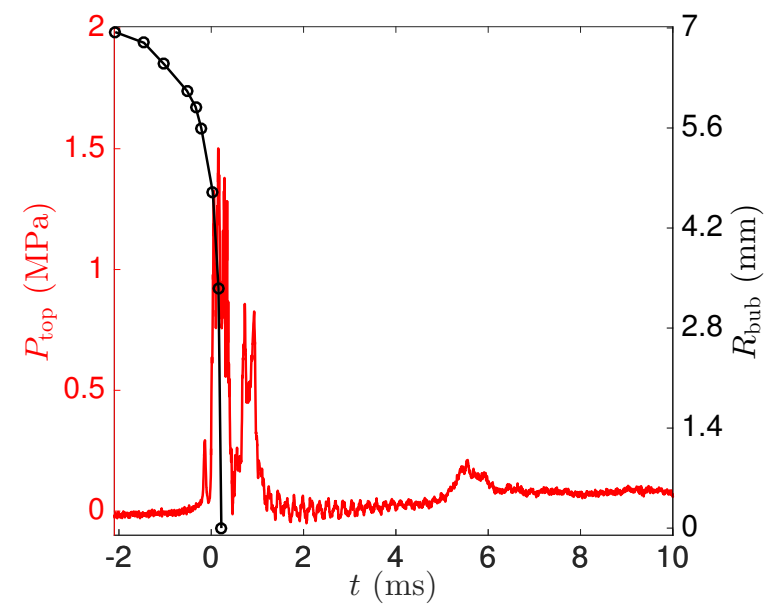

(a)

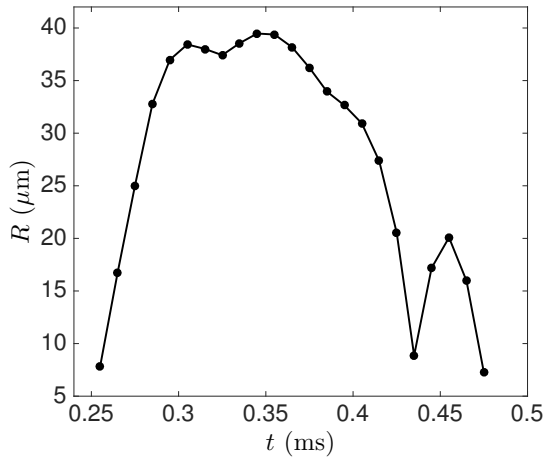

(b)

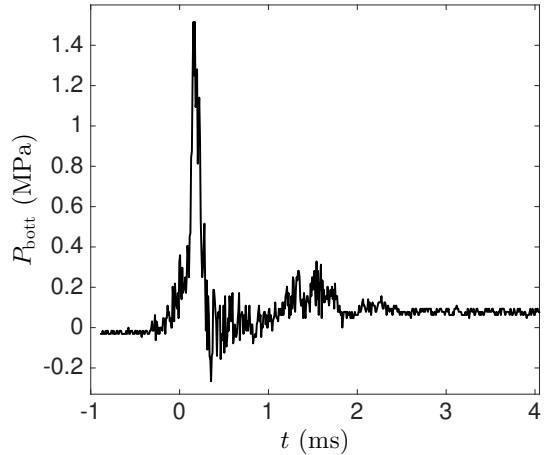

(c)

Figure 11: Top: Temporal evolution of the pressure under the piston (red line) and the bubble's size, $R_{\text {bub }}$ (black line) for a cavity with an initial radius of the order of the critical radius given by Eq. 77 $H_{0}=14 \mathrm{~mm}, d=0$ and $P_{\mathrm{top}, 0} / P_{\mathrm{vap}}=$ 1. Bottom left: Radius evolution of a bubble with an initial radius of few microns. Bottom right: Pressure at the bottom of the chamber for the experiment shown in Figure (b). 


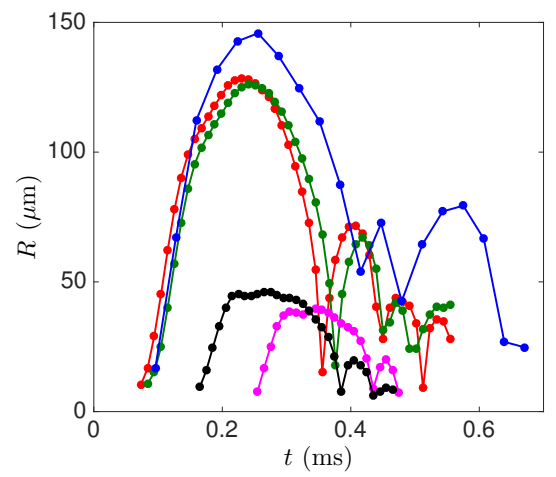

(a)

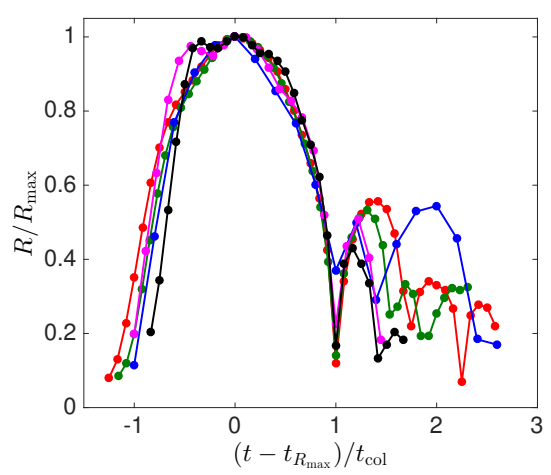

(b)

Figure 12: Left: Temporal evolution of the radii of various bubbles. Right: Dimensionless plot for the experimental data in (a). The various colors correspond to the evolution of bubbles for different shots. 


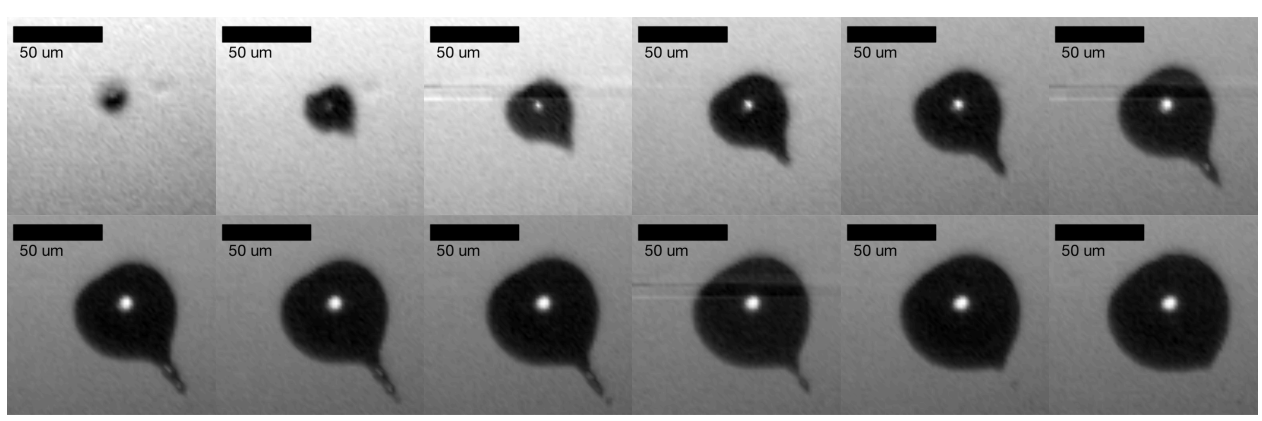

Figure 13: Snapshots during the collapse of a single bubble $\left(R_{\max }=79 \mu \mathrm{m}\right)$ generated by electrolysis. During the strong collapse a jet is generated that induces a strong translational motion. Interframe time, $7 \mu \mathrm{s}$.

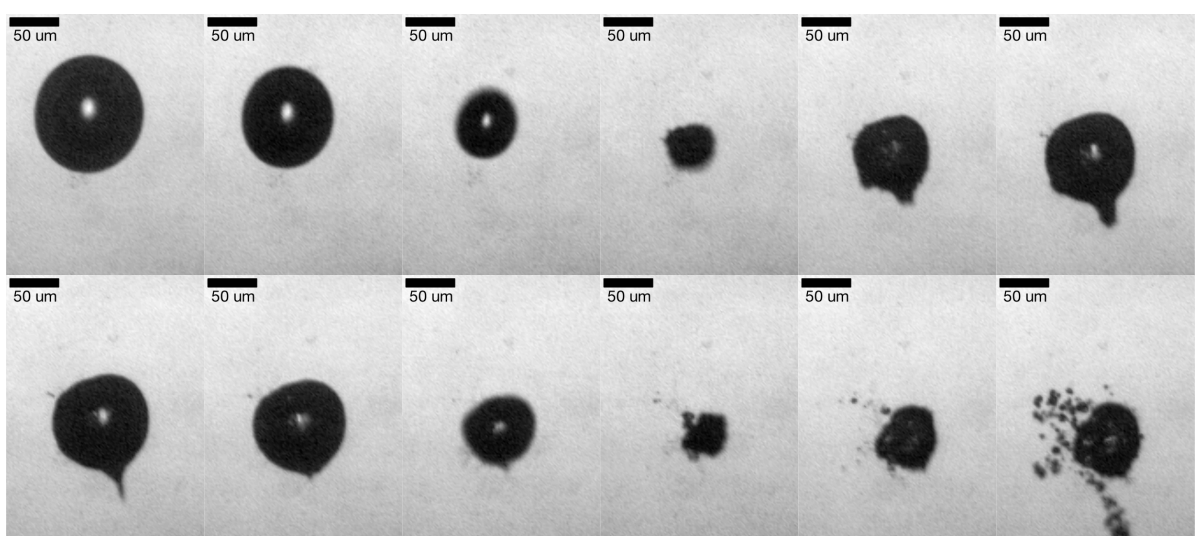

Figure 14: Snapshots during the collapse of a single bubble $\left(R_{\max }=62 \mu \mathrm{m}\right)$ generated by electrolysis. During the strong collapse a jet is generated that induces a strong translational motion. Interframe time, $15 \mu \mathrm{s}$. 


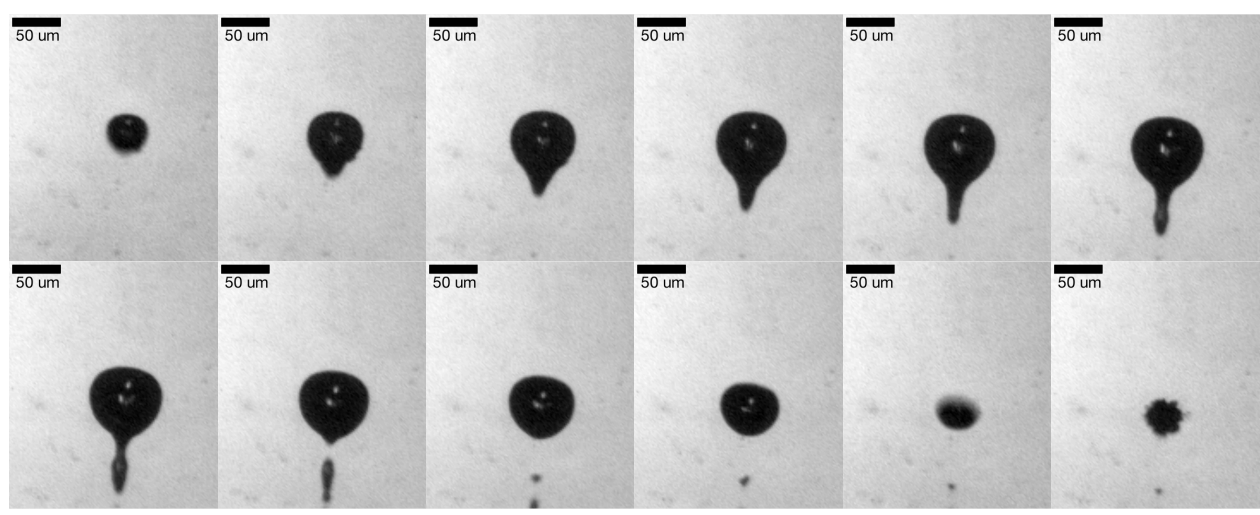

Figure 15: Snapshots during the collapse of a single bubble $\left(R_{\max }=154 \mu \mathrm{m}\right)$ generated by electrolysis. During the strong collapse a jet is generated that induces a strong translational motion. Interframe time, $10 \mu \mathrm{s}$. 

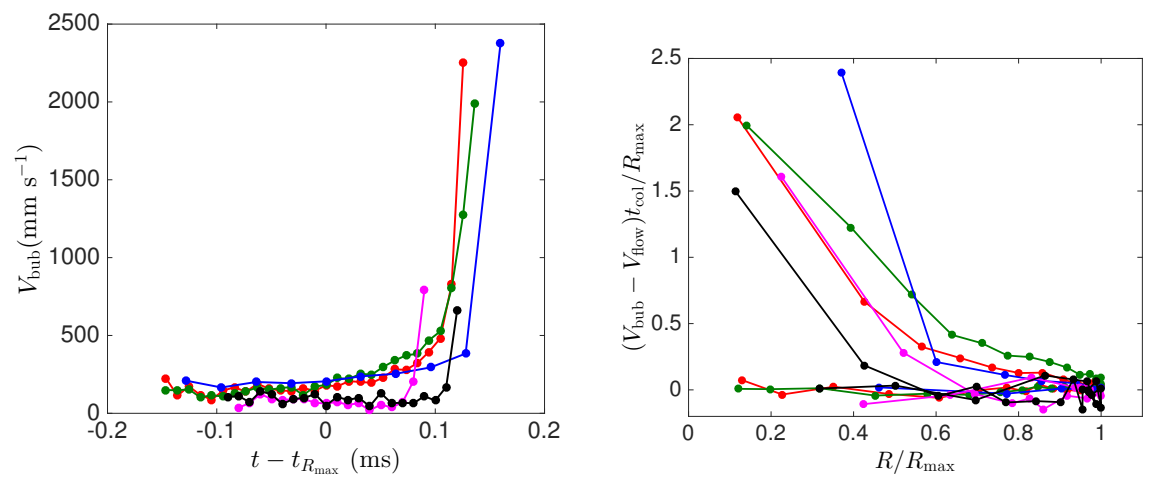

Figure 16: Left: Experimental data for the temporal evolution of the translational velocity of various bubbles. Right:Non-dimensional evolution of the translational velocity. Velocities up to $2 \mathrm{~m} \mathrm{~s}^{-1}$ are reached during the bubble collapse. 